\title{
A Study of a Fractional-Order Cholera Model
}

\author{
Mohammad Javidi ${ }^{1, *}$ and Bashir Ahmad ${ }^{2, *}$ \\ ${ }^{1}$ Faculty of Mathematical Sciences, University of Tabriz, Tabriz, Iran \\ ${ }^{2}$ Department of Mathematics, Faculty of Science, King Abdulaziz University, P.O. Box 80203, Jeddah, 21589, Saudi Arabia
}

Received: 16 Aug. 2013, Revised: 13 Nov. 2013, Accepted: 14 Nov. 2013

Published online: 1 Sep. 2014

\begin{abstract}
In this work, we investigate the dynamical behavior of a fractional order cholera model. All the feasible equilibria for the system are obtained and the conditions for the existence of interior equilibrium are determined. Local stability analysis of the cholera model is studied by using the fractional Routh-Hurwitz stability conditions. Our results indicate the potential of fractional-order cholera models to cope with modern epidemics.
\end{abstract}

Keywords: Cholera model, stability, numerical simulation, fractional order, dynamical system

\section{Introduction}

Cholera is an acute intestinal infection caused by ingestion of food or water contaminated with the bacterium Vibrio cholerae. It has a short incubation period, from less than one day to five days, and produces an enterotoxin that causes a copious, painless, watery diarrhoea that can quickly lead to severe dehydration and death if treatment is not promptly given. Vomiting also occurs in most patients. Cholera is an ancient disease that continues to cause epidemic and pandemic infection despite ongoing efforts to limit its spread ([1]-[7]). Historically, six out of the seven cholera pandemics have swept the globe since 1816 [8,9]. Most recently, the seventh pandemic started from Indonesia in 1961, spread into Europe, South Pacific and Japan in the late 1970s, reached South America in 1990s, and has continued (though much diminished) to the present. The last few years have witnessed many cholera outbreaks in developing countries, including Liberia (2002), Mali (2003), Senegal and Chad (2004), West Africa (2005), Angola and Sudan (2006), India (2007), Iraq and Congo (2008), Zimbabwe (2008-2009), Vietnam (2009), Nigeria, Central Africa, Pakistan and Haiti (2010), Sierra Leone (2012). Every year there are an estimated 3 to 5 million cholera cases and 100000 to 120000 deaths due to cholera $[9,10]$. Particularly, cholera represents a significant public health burden to developing countries and cholera continues receiving worldwide attention. Cholera is an extremely virulent disease. It affects both children and adults and can kill within hours. About 75\% of people infected with Vibrio cholerae do not develop any symptoms, although the bacteria are present in their faeces for 7-14 days after infection and are shed back into the environment, potentially infecting other people.

Many mathematical models have been proposed to investigate the complex epidemic and endemic behavior of cholera. The earliest mathematical model was proposed by Capasso and Paveri-Fontana [11] to study a cholera epidemic occurred in the Mediterranean in 1973. Codeco [12] in 2001 extended the work in [11] and explicitly accounted for the role of the aquatic reservoir in cholera dynamics. Using similar non-linear incidence in Codeos model, Hartley et al. [13] incorporated a hyper-infective stage of V. cholerae in 2006. This model emphasizes the stage of explosive infectivity of V. cholerae, based on the laboratory measurements that freshly shed V. cholerae from human intestines outcompeted other V. cholerae by as much as 700-fold for the first few hours in the environment [1,5]. Recently, Mukandavire et al. [14] proposed a model to estimate the reproduction number for the 2008-2009 cholera outbreak in Zimbabwe. Their model includes both environment-to-human and human-to-human transmission pathways. In 2010, Tien and Earn [15] published a water-borne disease model which also included the dual transmission pathways, with bilinear incidence rates employed for both the environment-to-human and human-to-human infection routes. Jensen et al. [16] proposed a mathematical model

\footnotetext{
*Corresponding author e-mail: mo_javidi@yahoo.com, bashirahmad_qau@yahoo.com
} 
to study how lytic bacteriophage specific for V. cholerae affects cholera outbreaks. In [17], the authors proposed a new and unified deterministic model that incorporates a general incidence rate and a general formulation of the pathogen concentration to analyse the dynamics of cholera. This work unifies many existing cholera models proposed by different authors. In [18], the authors proposed global stability analysis for several deterministic cholera epidemic models. These models, incorporating both human population and pathogen $\mathrm{V}$. cholerae concentration, constitute four-dimensional non-linear autonomous systems where the classical Poincar-Bendixson theory is not applicable.

Fractional-order differentiation is regarded as the generalization of classical integer-order differentiation to real or complex orders. There has been much interest in developing the theoretical analysis and numerical methods for fractional differential equations as fractional calculus is found to be a valuable tool in various fields of science and engineering. Indeed, we can find numerous applications in polymer rheology, regular variation in thermodynamics, biophysics, blood flow phenomena, aerodynamics, electro-dynamics of complex medium, viscoelasticity, Bode analysis of feedback amplifiers, capacitor theory, electrical circuits, electro-analytical chemistry, biology, control theory, fitting of experimental data, etc. ([19]-[21]). For some recent work on fractional differential equations and inclusions, see ([22]-[34]) and the references therein.

Recently, several investigators have studied the qualitative properties and numerical solutions of fractional-order biological models, for instance, see [35]. It has been mainly due to the reason that fractional-order equations are naturally related to systems with memory which exists in most biological systems. Also they are closely related to fractals which are abundant in biological systems. Yan and Kou [36] investigated stability properties of fractional-order differential equations and applied their results to analyze the stability of the equilibria for the model of HIV-1 infection.

In [37], the existence and uniqueness of solutions, stability of equilibria and numerical solutions for the fractional-order predator-prey model and rabies model were investigated. In [38], stability properties for a fractional-order model of nonlocal epidemics were studied and the results were found to be relevant to foot-and-mouth disease, SARS and avian flu. In addition, Ding and Ye have also introduced some kinds of models for HIV infection and discussed the stability of equilibria for the corresponding systems $[39,40]$.

The objective of this paper is to investigate a fractional-order cholera model by means of an efficient numerical method, based on an idea of transforming the given model to a system of ordinary differential equations of integer order. All the feasible equilibria for the system are discussed. The conditions ensuring the existence of interior equilibrium are also given. Local stability analysis of the cholera model is carried out by applying the fractional Routh-Hurwitz criterion. Our results show the worth of fractional-order cholera models to represent modern epidemics.

The paper is organized as follows. First of all, we describe our model. Section 3 contains some preliminary concepts. The stability for equilibria of the system is discussed in Section 4. In Section 5, the given model is studied numerically and the graphical results are presented in Section 6.

\section{The Model}

We consider the fractional-order Codeco model involving Caputo derivative given by [12]:

$$
\begin{aligned}
& \left\{\begin{array}{l}
\frac{d^{\alpha} S}{d t^{\alpha}}=n(H-S)-a \frac{S B}{K+B}, \\
\frac{d^{\alpha} I}{d t^{\alpha}}=a \frac{S B}{K+B}-r I, \\
\frac{d^{\alpha} B}{d t^{\alpha}}=B(n b-m b)+e I, \quad n b<m b,
\end{array}\right. \\
& S(\delta)=S_{0}, \quad I(\delta)=I_{0}>0, \quad B(\delta)=B_{0}>0,
\end{aligned}
$$

where the symbols appearing in this model are listed in Table 1. The first equation of (1) describes the dynamics of susceptibles in a community of constant size $\mathrm{H}$. Susceptible individuals are renewed at a rate $n$. Renewal may occur as result of birth, immigration and/or loss of acquired immunity (cholera apparently does not confer life-long immunity). Susceptible people becomes infected at a rate $a \frac{B}{K+B}$, where $a$ is the rate of contact with untreated water and $\frac{B}{K+B}$ is the probability of a person to catch cholera. Probability of catching cholera depends on the concentration of $V$. cholerae in the consumed water.

Table 1 . Symbols used in the model

\begin{tabular}{cc}
\hline Symbol & Description \\
\hline State Variables & number of susceptibles \\
$\mathrm{S}$ & number of infected \\
$\mathrm{I}$ & concentration of toxigenic V. cholerae in water (cells $/ \mathrm{ml}$ ) \\
$\mathrm{B}$ & total human population \\
$\begin{array}{c}\text { Parameters } \\
\mathrm{H}\end{array}$ & Human birth and death rates (day-1) \\
$\mathrm{n}$ & rate of exposure to contaminated water (day-1) \\
$\mathrm{a}$ & concentration of V. cholerae in water that yields $50 \%$ \\
$\mathrm{~K}$ & chance of catching cholera (cells/ml) \\
$\mathrm{r}$ & rate at which people recover from cholera (day-1) \\
$\mathrm{nb}$ & growth rate of V. cholerae in the aquatic environment (day-1) \\
$\mathrm{mb}$ & loss rate of V. cholerae in the aquatic environment (day-1) \\
$\mathrm{e}$ & contribution of each infected person to the population of \\
& V. cholerae in the aquatic environment (cell/ml day-1 person-1) \\
\hline
\end{tabular}

\section{Preliminaries}

The Riemann-Liouville fractional integral operator of order $\alpha>0$, of function $f \in L^{1}\left(\mathbb{R}^{+}\right)$is defined as

$I_{0}^{\alpha} f(t)=\frac{1}{\Gamma(\alpha)} \int_{0}^{t}(t-s)^{\alpha-1} f(s) d s$,

where $\Gamma(\cdot)$ is the Euler gamma function. 
The Riemann-Liouville and Caputo fractional derivative of order $\alpha>0, n-1<\alpha<n, n \in \mathbb{N}$ for a given continuous function $f$ are defined by

$D_{t}^{\alpha} f(t)=\frac{1}{\Gamma(n-\alpha)}\left(\frac{d}{d t}\right)^{n} \int_{a}^{t}(t-s)^{n-\alpha-1} f(s) d s$

$D_{t_{0}}^{\alpha} f(t)=\frac{1}{\Gamma(n-\alpha)} \int_{a}^{t} \frac{f^{(n)}(s)}{(t-s)^{\alpha+1-n}} d s$

In case of Caputo derivative, the function $f \in A C^{n-1}$. The initial value problem related to the above defnition is

$$
\left\{\begin{array}{l}
D^{\alpha} x(t)=f(t, x(t)), \\
\left.x(t)\right|_{t=0^{+}}=x_{0},
\end{array}\right.
$$

where $0<\alpha<1$ and $D^{\alpha}=D_{0}^{\alpha}$.

Now, we recall some stability theorems on fractionalorder systems.

Theorem 1 ([41]). The following autonomous system:

$$
\frac{d^{\alpha} x}{d t^{\alpha}}=A x, \quad x(0)=x_{0},
$$

with $0<\alpha \leq 1, x \in \mathbb{R}^{n}$ and $A \in \mathbb{R}^{n \times n}$, is asymptotically stable if and only if $|\arg (\lambda)|>\frac{\alpha \pi}{2}$ is satisfied for all eigenvalues of matrix $A$. Also, this system is stable if and only if $|\arg (\lambda)| \geq \frac{\alpha \pi}{2}$ is satisfied for all eigenvalues of matrix $A$ with those critical eigenvalues satisfying $|\arg (\lambda)|=\frac{\alpha \pi}{2}$ and having geometric multiplicity of one. The geometric multiplicity of an eigenvalue $\lambda$ of the matrix $A$ is the dimension of the subspace of vectors $v$ for which $A v=\lambda v$.

Theorem 2 ([42]). Consider the following commensurate fractional-order system:

$$
\frac{d^{\alpha} x}{d t^{\alpha}}=f(x), \quad x(0)=x_{0}
$$

with $0<\alpha \leq 1$ and $x \in \mathbb{R}^{n}$. The equilibrium points of system (4) are calculated by solving the equation: $f(x)=0$. These points are locally asymptotically stable if all eigenvalues $\lambda_{i}$ of the Jacobian matrix $J=\frac{\partial f}{\partial x}$ evaluated at the equilibrium points satisfy: $\left|\arg \left(\lambda_{i}\right)\right|>\frac{\alpha \pi}{2}$.

\section{Stability of equilibrium}

In this section, we analyze model (1) by finding its equilibria and studying their stability. Steady states of the model satisfy the following equations:

$$
\frac{d^{\alpha} S}{d t^{\alpha}}=0, \frac{d^{\alpha} I}{d t^{\alpha}}=0, \frac{d^{\alpha} B}{d t^{\alpha}}=0 .
$$

(5) has a trivial equilibrium $E_{0}=(H, 0,0)$. Let $R_{0}=\frac{a e H}{r K(m b-n b)}$ be the basic reproduction number. If
$R_{0}>1$ then (5) has a nontrivial equilibrium $E_{1}=(\bar{S}, \bar{I}, \bar{B})$, where

$$
\begin{aligned}
& \bar{S}=\frac{r K(m b-n b)+n e H}{e(n+a)}=\frac{H\left(a+R_{0}\right)}{R_{0}(a+n)}, \\
& \bar{B}=\frac{n(a e H-r K(m b-n b)}{e r(n+a)}=\frac{n K(m b-n b)\left(R_{0}-1\right)}{e(n+a)}, \\
& \bar{I}=\frac{(m b-n b) \bar{B}}{e}=\frac{n K(m b-n b)^{2}\left(R_{0}-1\right)}{e^{2}(n+a)} .
\end{aligned}
$$

To investigate the local behavior of system (1) about each of the equilibrium points, the Jacobian matrix $\mathrm{J}$ of the equilibrium point $E=(S, I, B)$ is computed as

$$
J(E)=\left(\begin{array}{ccc}
-n-\frac{a B}{K+B} & 0 & -\frac{a S K}{(K+B)^{2}} \\
\frac{a B}{K+B} & -r & \frac{a S K}{(K+B)^{2}} \\
0 & e & -(\mathrm{nb}-\mathrm{mb})
\end{array}\right)
$$

Now we consider the asymptotically stability of system (1) at the equilibrium point $E_{0}$. The equilibrium point $E_{0}$ is asymptotically stable if $R_{0}<1$.

The Jacobian matrix of (1) at equilibrium point $E_{0}$ is

$$
J\left(E_{0}\right)=\left(\begin{array}{ccc}
-n & 0 & -\frac{a H}{K} \\
0 & -r & \frac{a H}{K} \\
0 & e & (\mathrm{nb}-\mathrm{mb})
\end{array}\right)
$$

with the characteristic equation

$P(\lambda)=\lambda^{3}+b_{1} \lambda^{2}+b_{2} \lambda+b_{3}=0$

where

$$
b_{1}=m b-n b+n+r
$$

$$
\begin{aligned}
b_{2} & =(m b-n b)(n+r)+n r-\frac{e a H}{K} \\
& =n(m b-n b+r)+r(m b-n b)\left(1-R_{0}\right), \\
b_{3} & =-\frac{e a H}{K}+n r(m b-n b) \\
& =r n(m b-n b)\left(1-R_{0}\right),
\end{aligned}
$$$$
b_{1} b_{2}-b_{3}=n(m b-n b+n+r)(m b-n b+r)
$$$$
+(m b-n b+r) r(m b-n b)\left(1-R_{0}\right) .
$$

Therefore, the eigenvalues corresponding to the equilibrium $E_{0}$ are

$$
\begin{aligned}
& \lambda_{1}=-n, \\
& \lambda_{2,3}=\frac{-(m b-n b+r) \pm \sqrt{(m b-n b+r)^{2}+4 \frac{e a H}{K}}}{2} .
\end{aligned}
$$


Clearly, if $R_{0}<1$, then $\lambda_{1}, \lambda_{2}, \lambda_{3}<0$.

Let $D(P)$ denote the discriminant of a polynomial $P(\lambda)=$ $\lambda^{3}+b_{1} \lambda^{2}+b_{2} \lambda+b_{3}$. Then

$$
D(P)=18 b_{1} b_{2} b_{3}+\left(b_{1} b_{2}\right)^{2}-4 b_{3} b_{1}^{3}-4 b_{2}^{3}-27 b_{3}^{3} .
$$

The equilibrium point $E_{1}$ is asymptotically stable if one of the following conditions holds for polynomial $P$ and $D(P)$ :

(i) $D(P)>0, b_{1}>0, b_{3}>0$ and $b_{1} b_{2}>b_{3}$.

(ii) $D(P)<0, b_{1} \geq 0, b_{2} \geq 0, b_{3}>0$ and $\alpha<\frac{2}{3}$.

The Jacobian matrix of (1) at equilibrium point $E_{1}$ is

$$
J\left(E_{1}\right)=\left(\begin{array}{ccc}
-n-\frac{a \bar{B}}{K+\bar{B}} & 0 & -\frac{r(\mathrm{mb}-\mathrm{nb})}{e} \frac{K}{(K+\bar{B})} \\
\frac{a \bar{B}}{K+\bar{B}} & -r & \frac{r(\mathrm{mb}-\mathrm{nb})}{e} \frac{K}{(K+\bar{B})} \\
0 & e & -(\mathrm{nb}-\mathrm{mb})
\end{array}\right)
$$

with the characteristic equation

$P(\lambda)=\lambda^{3}+b_{1} \lambda^{2}+b_{2} \lambda+b_{3}=0$,

where

$b_{1}=m b-n b+n+r+\frac{a \bar{B}}{K+\bar{B}}$,

$b_{2}=r\left(n+\frac{a \bar{B}}{K+\bar{B}}\right)+(m b-n b)\left(r+n+\frac{a \bar{B}}{K+\bar{B}}\right)-\frac{r K(m b-n b)}{K+\bar{B}}$,

$b_{3}=\left(r(m b-n b)-\frac{r(m b-n b) K}{K+\bar{B}}\right)\left(n+\frac{a \bar{B}}{K+\bar{B}}\right)+\frac{r(m b-n b) K a \bar{B}}{K+\bar{B}}$.

Observe that $b_{1}>0$. Manipulating $b_{2}$ and $b_{3}$, we have

$b_{2}=n(m b-n b)+\frac{r K(m b-n b)+[r(n+m b-n b)+a(m b-n b+r)] \bar{B}}{K+\bar{B}}$,

$b_{3}=\frac{C_{0}+C_{1} \bar{B}+C_{2} \bar{B}^{2}}{(K+\bar{B})^{2}}$.

where

$C_{0}=r n^{2} K^{2}+r^{2} n K^{2}+K(m b-n b) r^{2} n$,

$C_{1}=r n K a+n K r^{2}+r n^{2} K+K r(n+r+m b-n b)(n+m b-n b)$

$+K a(m b-n b+r)(n+r+m b-n b)+r^{2} n(m b-n b)$,

$C_{2}=(r+m b-n b)[r(n+m b-n b)+a(m b-n b+r)]+(a+n)[n r+a(m b-n b+r)]$.

It is clear that $C_{0}, C_{1}, C_{2}>0$. Therefore $b_{2}, b_{3}>0$.

\section{Numerical method}

Here, we shall use a numerical method introduced by Atanackovic and Stankovic $[43,44]$ to solve the fractional-order nonlinear system (1). In [43], it was shown that the fractional derivative of order $\alpha$ $(0<\alpha \leq 1)$ for a function $f(t)$ may be expressed as

$$
D^{\alpha} f(t)=\frac{1}{\Gamma(2-\alpha)}\left\{\frac{f^{(1)}(t)}{t^{\alpha-1}}\left[1+\sum_{p=1}^{\infty} \frac{\Gamma(p-1+\alpha)}{\Gamma(\alpha-1) p !}\right]\right.
$$

$\left.-\left[\frac{\alpha-1}{t^{\alpha}} f(t)+\sum_{p=2}^{\infty} \frac{\Gamma(p-1+\alpha)}{\Gamma(\alpha-1)(p-1) !}\left(\frac{f(t)}{t^{\alpha}}+\frac{V_{p}(f)(t)}{t^{p-1+\alpha}}\right)\right]\right\}$,

where

$V_{p}(f)(t)=-(p-1) \int_{0}^{t} \tau^{p-2} f(\tau) d \tau, p=2,3, \cdots$,

$\frac{d}{d t} V_{p}(f)=-(p-1) t^{p-2} f(t), p=2,3, \cdots$.

We approximate $D^{\alpha} f(t)$ by using $M$ terms in sums appearing in (9) as follows

$$
D^{\alpha} f(t) \simeq \frac{1}{\Gamma(2-\alpha)}\left\{\frac{f^{(1)}(t)}{t^{\alpha-1}}\left[1+\sum_{p=1}^{M} \frac{\Gamma(p-1+\alpha)}{\Gamma(\alpha-1) p !}\right]\right.
$$

$\left.-\left[\frac{\alpha-1}{t^{\alpha}} f(t)+\sum_{p=2}^{M} \frac{\Gamma(p-1+\alpha)}{\Gamma(\alpha-1)(p-1) !}\left(\frac{f(t)}{t^{\alpha}}+\frac{V_{p}(f)(t)}{t^{p-1+\alpha}}\right)\right]\right\}$.

We can rewrite (12) as

$D^{\alpha} f(t) \simeq \Omega(\alpha, t, M) f^{(1)}(t)+\Phi(\alpha, t, M) f(t)+\sum_{p=2}^{M} A(\alpha, t, p) \frac{V_{p}(f)(t)}{t^{p-1+\alpha}}$

where

$$
\begin{gathered}
\Omega(\alpha, t, M)=\frac{1+\sum_{p=1}^{M} \frac{\Gamma(p-1+\alpha)}{\Gamma(\alpha-1) p !}}{\Gamma(2-\alpha) t^{\alpha-1}} \\
R(\alpha, t)=\frac{1-\alpha}{t^{\alpha} \Gamma(2-\alpha)}, A(\alpha, t, p)=-\frac{\Gamma(p-1+\alpha)}{\Gamma(2-\alpha) \Gamma(\alpha-1) p !} \\
\Phi(\alpha, t, M)=R(\alpha, t)+\sum_{p=2}^{M} \frac{A(\alpha, t, p)}{t^{\alpha}}
\end{gathered}
$$

We set

$\Theta_{1}(t)=S(t), \Theta_{M+1}(t)=I(t)$,

$\Theta_{2 M+1}(t)=B(t), \Theta_{p}(t)=V_{p}(S)(t)$,

$\Theta_{p+M}(t)=V_{p}(I)(t), \Theta_{2 M+p}(t)=V_{p}(B)(t)$,

for $p=2,3, \cdots$.

We can rewrite system (1) in the following form

$$
\begin{gathered}
\Omega(\alpha, t, M) \Theta_{1}^{\prime}(t)+\Phi(\alpha, t, M) \Theta_{1}(t)+\sum_{p=2}^{M} A(\alpha, t, p) \frac{\Theta_{p}(t)}{t^{p-1+\alpha}} \\
=n\left(H-\Theta_{1}(t)\right)-a \frac{\Theta_{1}(t) \Theta_{2 M+1}(t)}{K+\Theta_{2 M+1}(t)}, \\
\Omega(\alpha, t, M) \Theta_{M+1}^{\prime}(t)+\Phi(\alpha, t, M) \Theta_{M+1}(t)+\sum_{p=2}^{M} A(\alpha, t, p) \frac{\Theta_{M+p}(t)}{t^{p-1+\alpha}} \\
=a \frac{\Theta_{1}(t) \Theta_{2 M+1}(t)}{K+\Theta_{2 M+1}(t)}-r \Theta_{M+1}(t), \\
\Omega(\alpha, t, M) \Theta_{2 M+1}^{\prime}(t)+\Phi(\alpha, t, M) \Theta_{2 M+1}(t)+\sum_{p=2}^{M} A(\alpha, t, p) \frac{\Theta_{2 M+p}(t)}{t^{p-1+\alpha}} \\
=(n b-m b) \Theta_{2 M+1}+e \Theta_{M+1}(t),
\end{gathered}
$$


where

$$
\begin{gathered}
\Theta_{p}(t)=-(p-1) \int_{0}^{t} \tau^{p-2} \Theta_{1}(\tau) d \tau, \\
\Theta_{M+p}(t)=-(p-1) \int_{0}^{t} \tau^{p-2} \Theta_{M+1}(\tau) d \tau, \\
\Theta_{2 M+p}(t)=-(p-1) \int_{0}^{t} \tau^{p-2} \Theta_{2 M+1}(\tau) d \tau,
\end{gathered}
$$$$
p=2,3, \cdots, M \text {. }
$$

Finally (14) and (15) can be rewritten as

$$
\begin{gathered}
\Theta_{1}^{\prime}(t)=\frac{1}{\Omega(\alpha, t, M)}\left(n\left(H-\Theta_{1}(t)\right)-a \frac{\Theta_{1}(t) \Theta_{2 M+1}(t)}{K+\Theta_{2 M+1}(t)}\right) \\
\left.-\Phi(\alpha, t, M) \Theta_{1}(t)-\sum_{p=2}^{M} A(\alpha, t, p) \frac{\Theta_{p}(t)}{t^{p-1+\alpha}}\right), \\
\Theta_{p}^{\prime}(t)=-(p-1) t^{p-2} \Theta_{1}(t), p=2,3, \cdots, M, \\
\Theta_{M+1}^{\prime}(t)=\frac{1}{\Omega(\alpha, t, M)}\left(a \frac{\Theta_{1}(t) \Theta_{2 M+1}(t)}{K+\Theta_{2 M+1}(t)}-r \Theta_{M+1}(t)\right) \\
\left.-\Phi(\alpha, t, M) \Theta_{M+1}(t)-\sum_{p=2}^{M} A(\alpha, t, p) \frac{\Theta_{M+p}(t)}{t^{p-1+\alpha}}\right), \\
\Theta_{M+p}^{\prime}(t)=-(p-1) t^{p-2} \Theta_{M+1}(t), p=2,3, \cdots, M, \\
\Theta_{2 M+1}^{\prime}(t)=\frac{1}{\Omega(\alpha, t, M)}\left((n b-m b) \Theta_{2 M+1}+e \Theta_{M+1}(t)\right) \\
\Theta_{2 M+p}^{\prime}(t)=-(p-1) t^{p-2} \Theta_{2 M+1}(t), p=2,3, \cdots, M, \\
\left.-\Phi(\alpha, t, M) \Theta_{2 M+1}(t)-\sum_{p=2}^{M} A(\alpha, t, p) \frac{\Theta_{2 M+p}(t)}{t^{p-1+\alpha}}\right),
\end{gathered}
$$

with the following initial conditions

$$
\begin{aligned}
& \Theta_{1}(\delta)=S_{0}, \\
& \Theta_{p}(\delta)=-\frac{p-1}{2} \Delta t^{p-1} S_{0}, p=2,3, \cdots, M, \\
& \Theta_{M+1}(\delta)=I_{0}, \\
& \Theta_{M+p}(\delta)=-\frac{p-1}{2} \Delta t^{p-1} I_{0}, p=2,3, \cdots, M, \\
& \Theta_{2 M+1}(\delta)=B_{0}, \\
& \Theta_{2 M+p}(\delta)=\frac{p-1}{2} \Delta t^{p-1} B_{0}, p=2,3, \cdots, M .
\end{aligned}
$$

In the next section, we solve the system (16) with the initial conditions (17) by using the well known Runge-Kutta method of order fourth.

\section{Numerical Simulation and discussion}

To facilitate the interpretation of our mathematical results developed for the model (1) so far, we proceed to investigate it by numerical simulations. We solve the system (1) numerically by using the method proposed in the previous section. In all numerical runs, the solution has been approximated at $\delta=\Delta t=0.01, M=5, \eta=m b-n b$. We illustrate our numerical results by considering a variety of examples.

\section{Example 1.}

We consider the following set of parameters:

$H=1000, N=0.001, a=0.1, K=1000, r=0.4, \eta=$ $0.4, e=1$.

The stability of equilibria $E_{0}$ can be seen in Figs. 1-2, with the initial conditions $\left[S_{0}, I_{0}, B_{0}\right]=$ $[986,10,4],[974,20,6],[965,30,5],[932,60,8] \quad$ and simulation time $T=1200$ for $\alpha=0.95$ and $\alpha=0.99$ respectively. It is easy to compute that $R_{0}=0.6250<1$.

\section{Example 2.}

Let us choose a set of parameters:

$H=100, N=0.001, a=0.5, K=100, r=0.4, \eta=$ $0.02, e=1$,

and the initial conditions $\left[S_{0}, I_{0}, B_{0}\right]==[81,15,4],[69,25,6],[60,35,5],[32,60,8]$ with simulation time $T=1800$ for $\alpha=0.6<\frac{2}{3}$ and $\alpha=0.5<\frac{2}{3}$. The stability of equilibria $E_{1}=(1.7964,0.2455,12.2754)$ can be observed in Figs. $3-4$. One can easily find that $R_{0}=62.5>1, b_{1}=$ $0.4757>0, b_{2}=0.0243>0, b_{3}=4.3821 e-004>$ $0, b_{1} b_{2}-b_{3}=0.0111>0, D(P)=-2.1616 e-005$.

\section{Example 3.}

In this case, we consider the following set of parameters: 

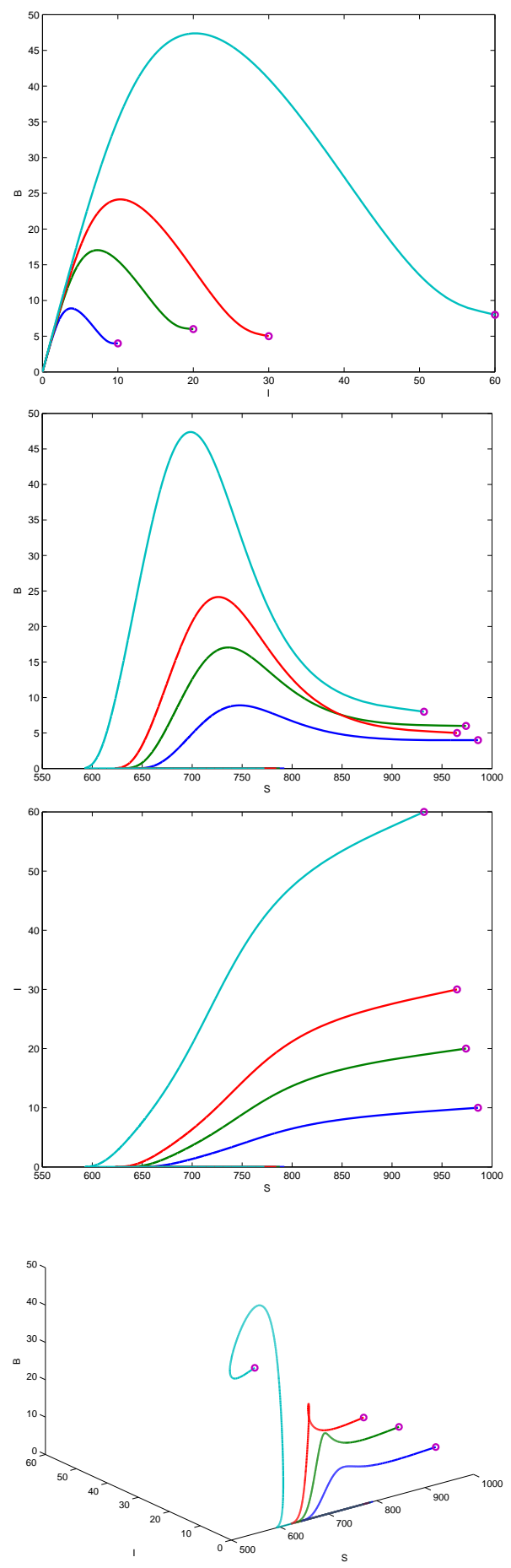

Fig. 1: Stability of the equilibria $E_{0}$. Consider the following choice of parametric values: $H=1000, N=0.001, a=0.1, K=$ $1000, r=0.4, \eta=0.4, e=1$. For $\alpha=0.95$ and $R_{0}=0.6250<1$.
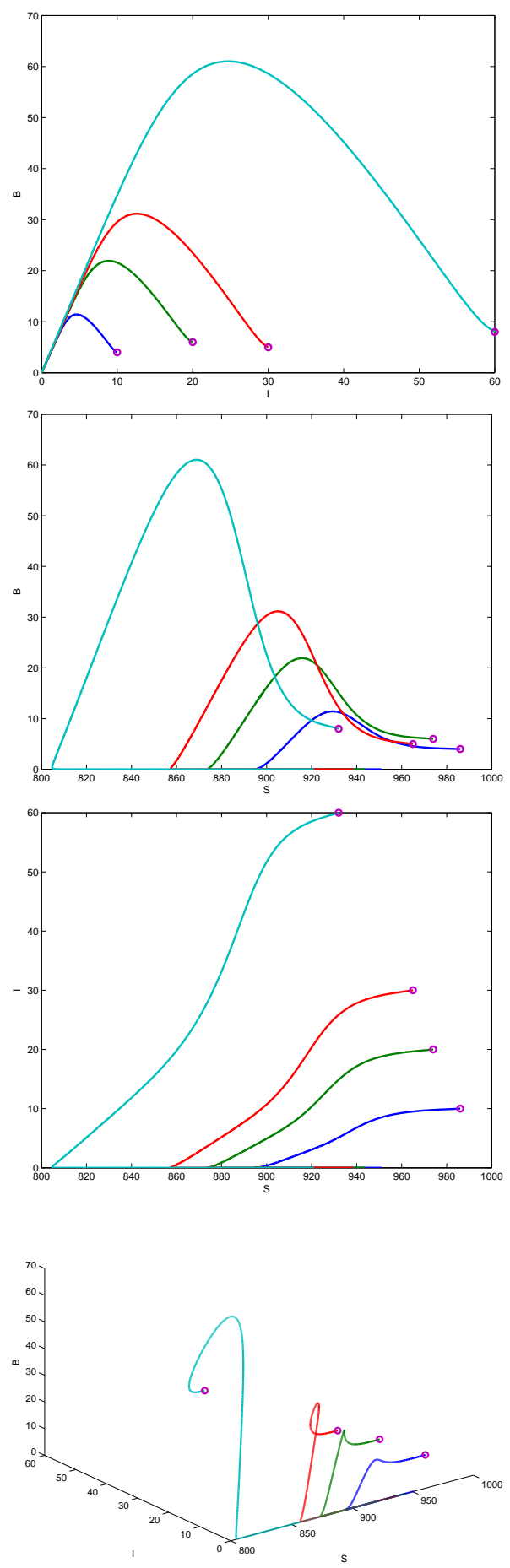

Fig. 2: Stability of the equilibria $E_{0}$. Consider the following choice of parametric values: $H=1000, N=0.001, a=0.1, K=$ $1000, r=0.4, \eta=0.4, e=1$. For $\alpha=0.99$ and $R_{0}=0.6250<1$. 

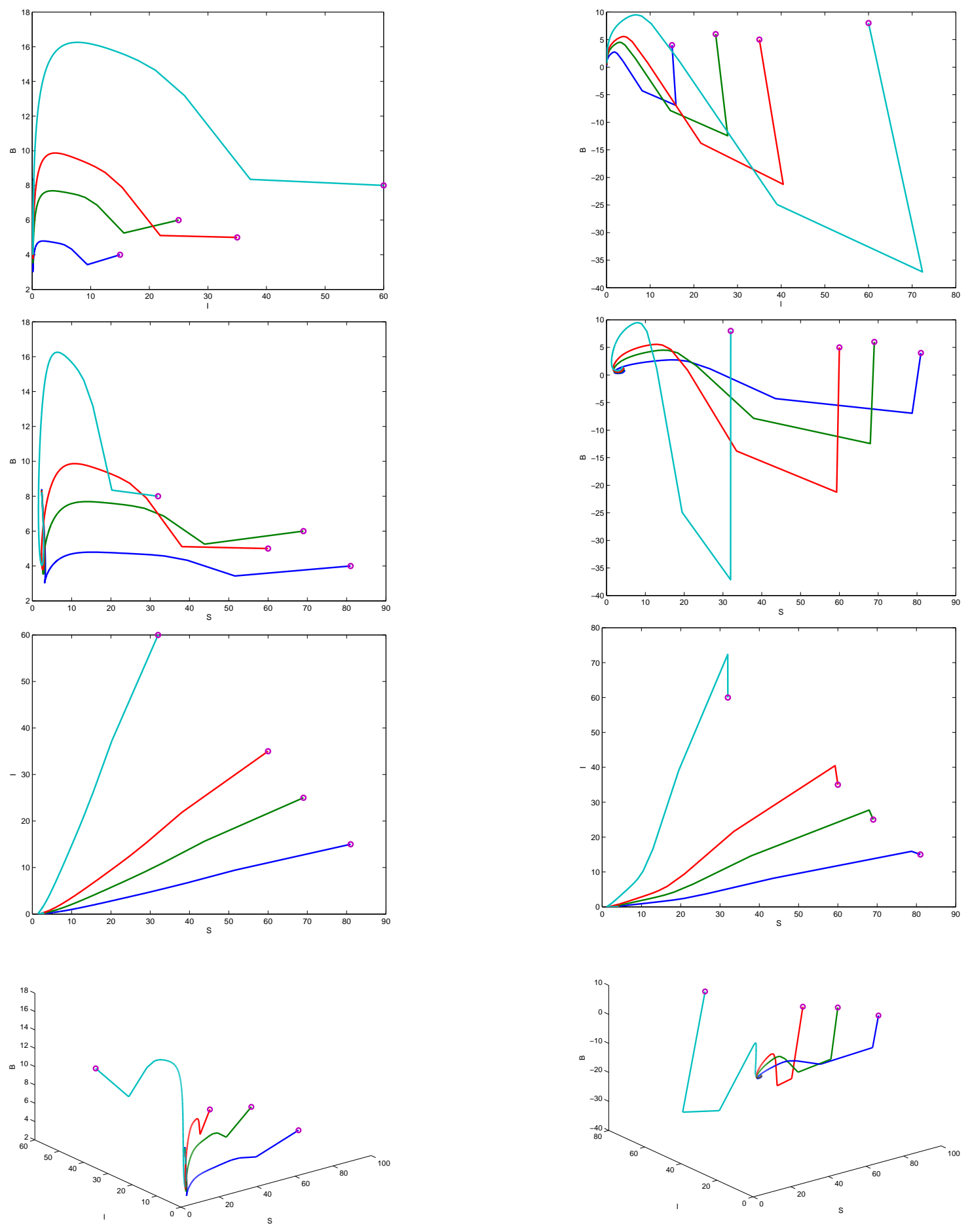

Fig. 3: Stability of the equilibria $E_{1}$. Consider the following choice of parametric values: $H=100, N=0.001, a=0.5, K=$ $100, r=0.4, \eta=0.02, e=1$. For $\alpha=0.6$ and $R_{0}=62.5$.

Fig. 4: Stability of the equilibria $E_{1}$. Consider the following choice of parametric values: $H=100, N=0.001, a=0.5, K=$ $100, r=0.4, \eta=0.02, e=1$. For $\alpha=0.5$ and $R_{0}=62.5$. 
$H=1000, N=0.001, a=0.7, K=1000, r=0.4, \eta=$ $0.02, e=1$.

with the initial conditions $\left[S_{0}, I_{0}, B_{0}\right]=$ $[986,155,25],[705,235,60],[475,435,50],[860,60,80]$ and simulation time $T=1000$ for $\alpha=0.95,0.9,0.8$ and $\alpha=0.7$. The stability of equilibria $E_{1}=(12.8388,2.4679,123.3951)$ is shown in Figs. 5-8. With the given data, we find that $R_{0}=87.5>1, b_{1}=$ $0.4979>0, b_{2}=0.0336>0, b_{3}=6.1599 e-004>$ $0, b_{1} b_{2}-b_{3}=0.0161>0, D(P)=9.4334 e-006$.

\section{Example 4.}

Consider the following values of parameters: $N=0.002, a=0.2, K=H, r=0.05, \eta=0.13, e=20$, and initial conditions: $\left[S_{0}, I_{0}, B_{0}\right]=[H-180,155,25]$ with simulation time: $T=250$ for $\alpha=0.9$ and $H=1000,3000,5000,7000$. It is easy to compute that $R_{0}=615.3846>1$. The numerical solution of (1) is shown by Fig. 9 .

\section{Conclusions}

In this paper, we have studied several features of a fractional-order cholera model. These features can be summarized as follows. (i) We present criteria for the existence of infected-free equilibria and concentration of toxigenic V. cholerae in water equilibria. (ii) Stability of the equilibria for the system (1) has been discussed in terms of the reproduction number $R_{0}=\frac{a e H}{r K(m b-n b)}$. Precisely, we have established the following facts: if $R_{0}<1$, then the equilibrium $E_{0}$ of system (1) is locally asymptotically stable for all $0<\alpha<1$; the equilibrium $E_{1}$ of system (1) is locally asymptotically stable if $R_{0}>0$. Also the stability analysis for the system (1) is carried out by applying the fractional Routh-Hurwitz method. (iii) The fractional-order model (1) is converted to a system of ordinary differential equations of integer-order and is then solved numerically by using the fourth order Runge-Kutta method. The graphical solutions are presented for several choices of the parameters and conditions involved in the model.

\section{Acknowledgement}

The authors are grateful to the anonymous referee for a careful checking of the details and for helpful comments that improved this paper.
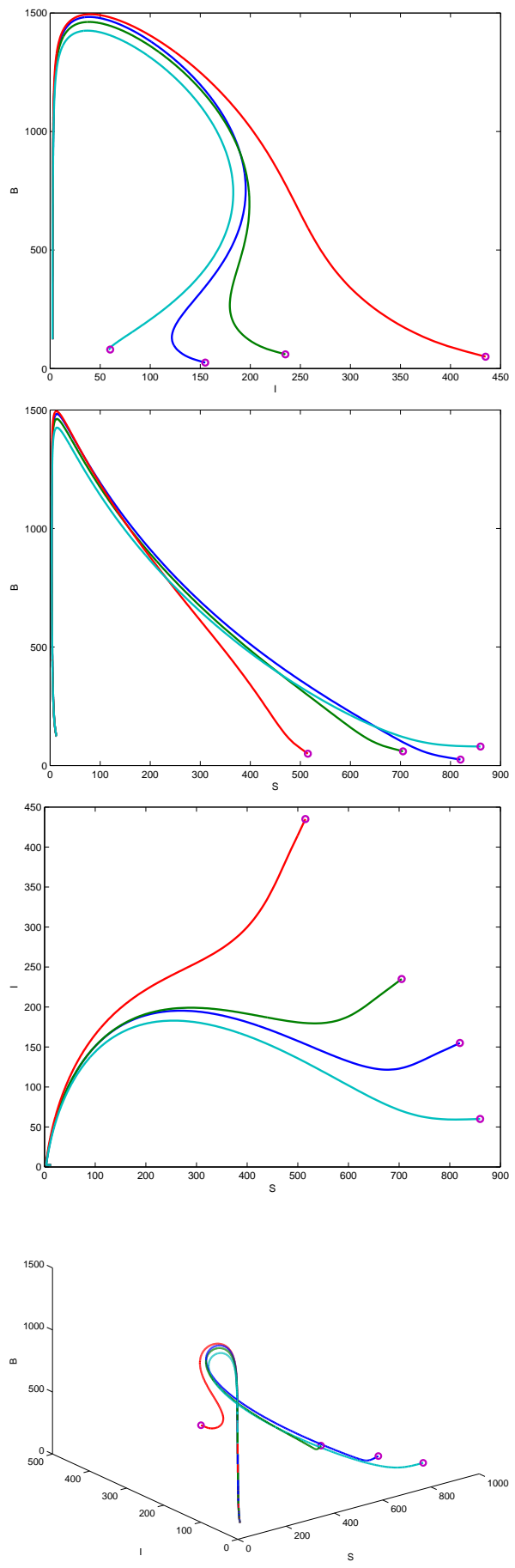

Fig. 5: Stability of the equilibria $E_{1}$. Consider the following choice of parametric values: $H=1000, N=0.001, a=0.7, K=$ $1000, r=0.4, \eta=0.02, e=1$. For $\alpha=0.95$ and $R_{0}=87.5$. 

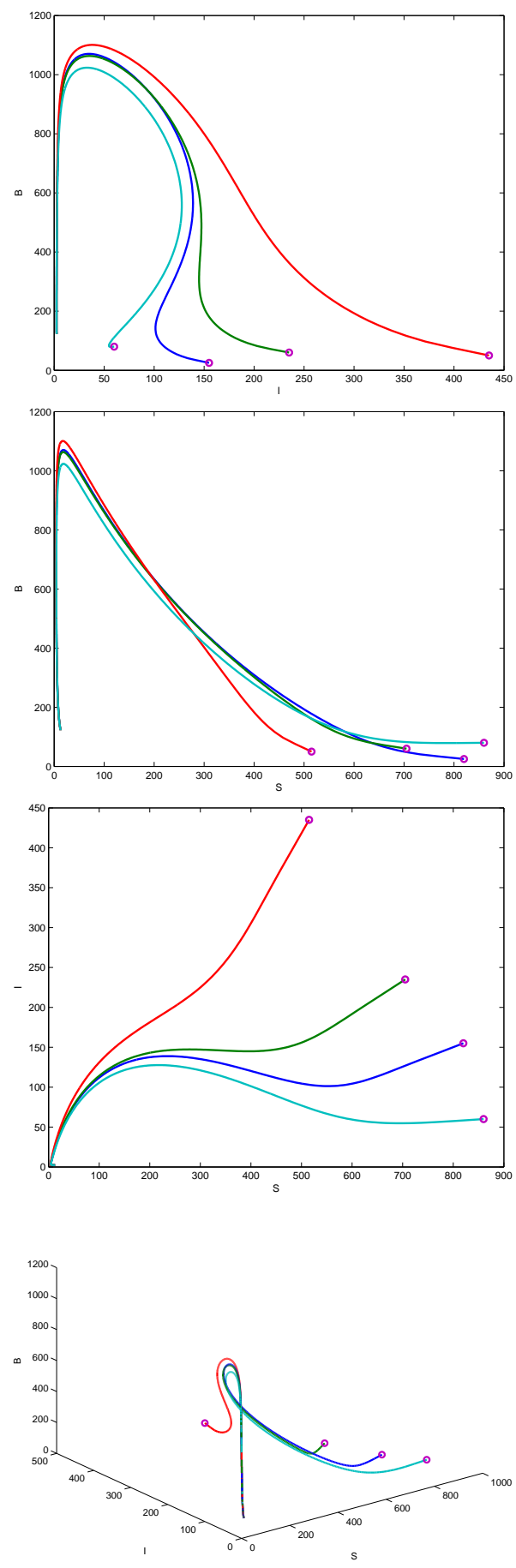

Fig. 6: Stability of the equilibria $E_{1}$. Consider the following choice of parametric values: $H=1000, N=0.001, a=0.7, K=$ $1000, r=0.4, \eta=0.02, e=1$. For $\alpha=0.9$ and $R_{0}=87.5$.
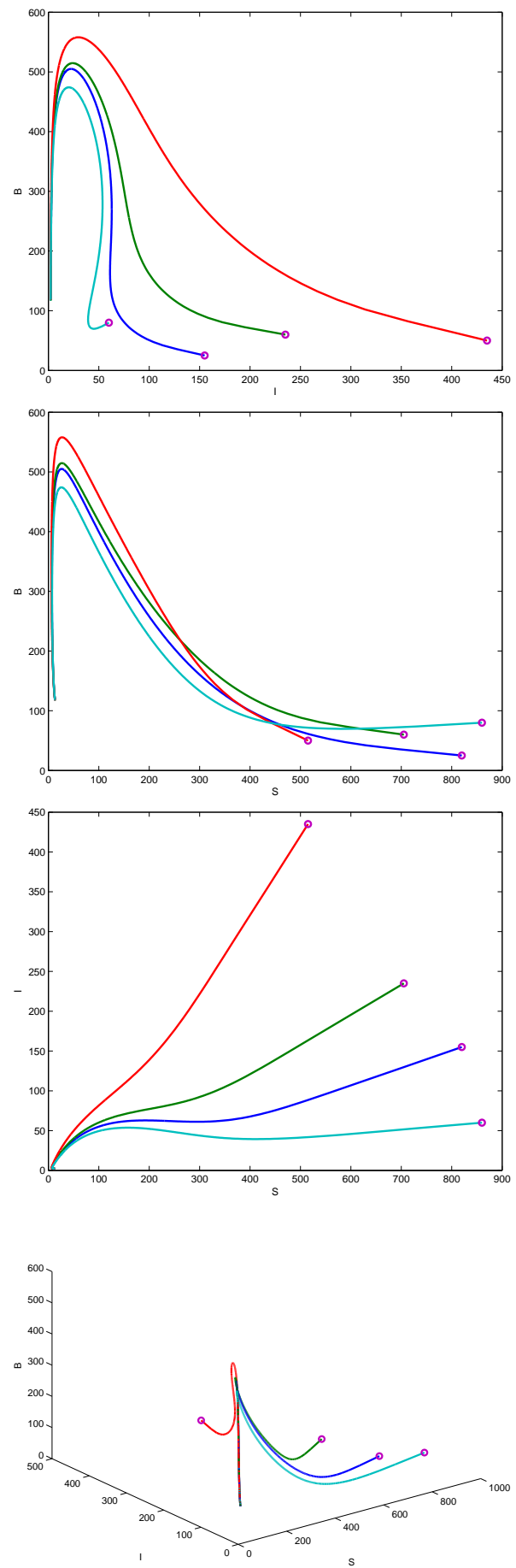

Fig. 7: Stability of the equilibria $E_{1}$. Consider the following choice of parametric values: $H=1000, N=0.001, a=0.7, K=$ $1000, r=0.4, \eta=0.02, e=1$. For $\alpha=0.8$ and $R_{0}=87.5$. 

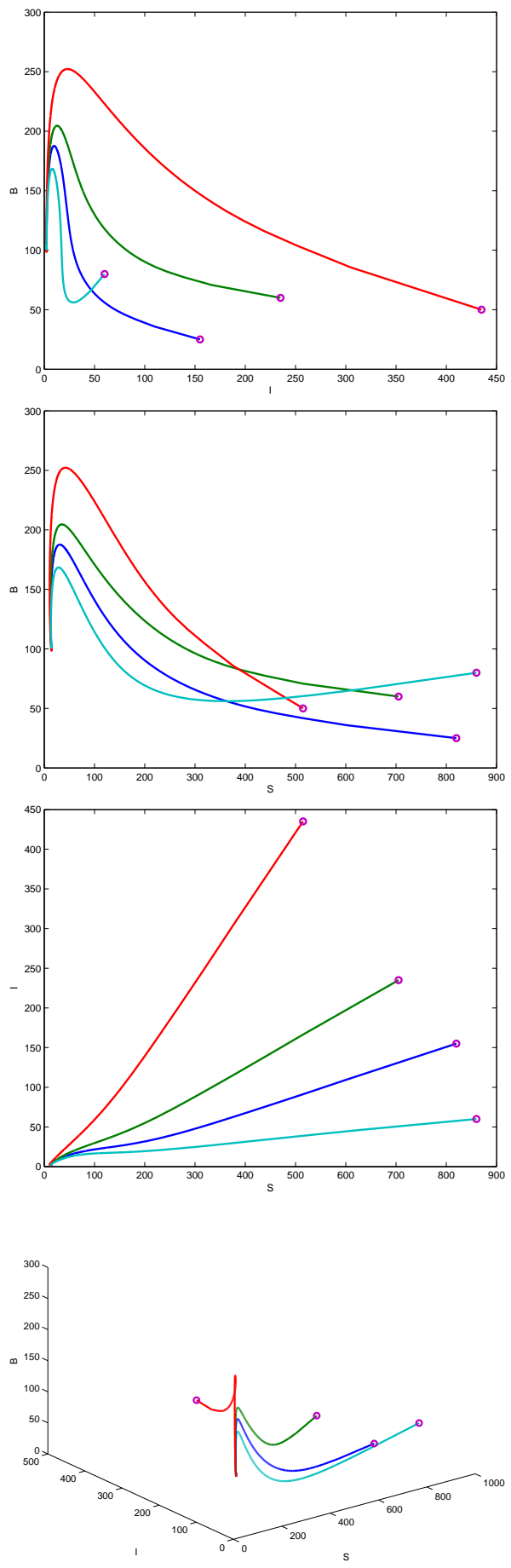

Fig. 8: Stability of the equilibria $E_{1}$. Consider the following choice of parametric values: $H=1000, N=0.001, a=0.7, K=$ $1000, r=0.4, \eta=0.02, e=1$. For $\alpha=0.7$ and $R_{0}=87.5$.
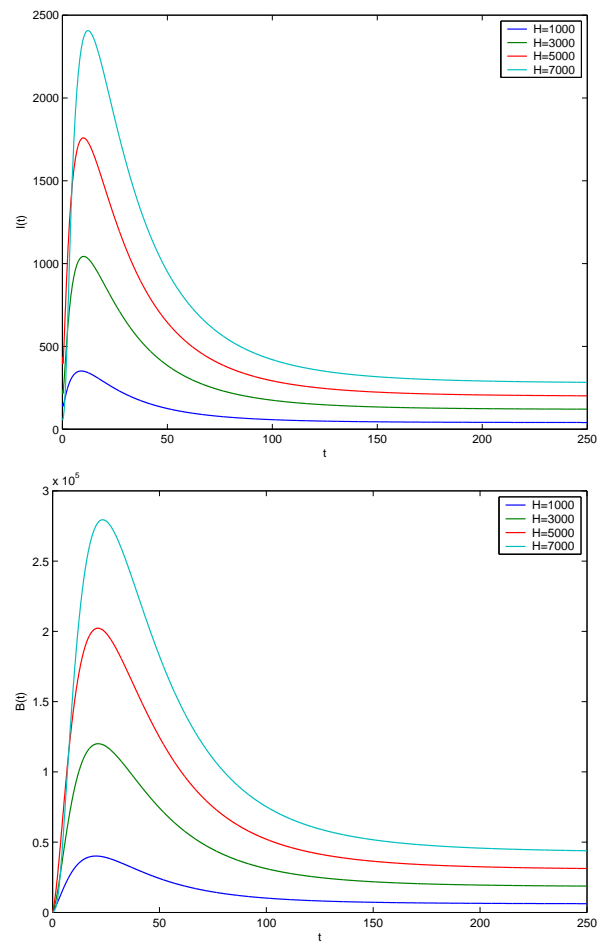

Fig. 9: Numerical solution of (1). Consider the following choice of parametric values: $N=0.002, a=0.2, K=H, r=$ $0.05, \eta=0.13, e=20$ For $\alpha=0.9, R_{0}=615.3846$ and $H=$ $1000,3000,5000,7000$.

\section{References}

[1] A. Alam, R.C. Larocque, J.B. Harris, et al., Hyperinfectivity of human-passaged Vibrio cholerae can be modeled by growth in the infant mouse, Infect. Immun., 73, 6674 (2005).

[2] M. Ghosh, P. Chandra, P. Sinha, J.B. Shukla, Modeling the spread of carrierdependent infectious diseases with environmental effect, Appl. Math. Comput., 152, 385 (2004).

[3] T.R. Hendrix, The pathophysiology of cholera, B. New York Acad. Med., 47, 1169 (1971).

[4] A.A. King, E.L. Lonides, M. Pascual, M.J. Bouma, Inapparent infections and cholera dynamics, Nature, 454, 877 (2008).

[5] D.S. Merrell, S.M. Butler, F. Qadri, et al., Host-induced epidemic spread of the cholera bacterium, Nature, 417, 642 (2002).

[6] E.J. Nelson, J.B. Harris, J.G. Morris, S.B. Calderwood, A. Camilli, Cholera transmission: the host, pathogen and bacteriophage dynamics, Nat. Rev. Microbiol., 7, 693 (2009).

[7] M. Pascual, M. Bouma, A. Dobson, Cholera and climate: revisiting the quantitative evidence, Microbes Infect., 4, 237 (2002).

[8] Center for Disease Control and Prevention web page: www.cdc.gov.

[9] World Health Organization web page: www.who.org.

[10] The Wikipedia: en.wikipedia.org. 
[11] V. Capasso, S.L. Paveri-Fontana, A mathematical model for the 1973 cholera epidemic in the european mediterranean region, Revue dpidmoligi et de sant Publiqu, 27, 121 (1979).

[12] C.T. Codeo, Endemic and epidemic dynamics of cholera: the role of the aquatic reservoir, BMC Infect. Dis., 1, 1 (2001).

[13] D.M. Hartley, J.G. Morris, D.L. Smith, Hyperinfectivity: a critical element in the ability of V. cholerae to cause epidemics, Plos Med., 3, 0063 (2006).

[14] Z. Mukandavire, S. Liao, J. Wang, H. Gaff, D.L. Smith, J.G. Morris Jr., Estimating the reproductive numbers for the 20082009 cholera outbreak in Zimbabwe, Proc. Natl. Acad. Sci. USA, 108, 8767-72 (2011).

[15] J.H. Tien, D.J.D. Earn, Multiple transmission pathways and disease dynamics in a waterborne pathogen model, B. Math. Biol., 72, 1502 (2010).

[16] M. Jensen, S.M. Faruque, J.J. Mekalanos, B. Levin, Modeling the role of bacteriophage in the control of cholera outbreaks, Proceedings of the National Academy of Sciences, 103, 4652 (2006).

[17] J. Wang, S. Liao, A generalized cholera model and epidemic/endemic analysis, J. Biol. Dynam., 6, 568-589 (2012).

[18] J.P. Tian, J. Wang, Global stability for cholera epidemic models, Math. Biosci., 232, 31-41 (2011).

[19] I. Podlubny, Fractional Differential Equations, Academic Press, San Diego, (1999).

[20] A.A. Kilbas, H.M. Srivastava, J.J. Trujillo, Theory and Applications of Fractional Differential Equations, in: North-Holland Mathematics Studies, Elsevier Science B.V, Amsterdam, 204, (2006).

[21] J. Sabatier, O.P. Agrawal, J.A.T. Machado (Eds.), Advances in Fractional Calculus: Theoretical Developments and Applications in Physics and Engineering, Springer, Dordrecht, (2007).

[22] A.M.A. El-Sayed, A.E.M. El-Mesiry, H.A.A. El-Saka, On the fractional-order logistic equation, Appl. Math. Lett., 20, 817-823 (2007).

[23] M. Benchohra, J. Henderson, S.K. Ntouyas, A. Ouahab, Existence results for fractional order functional differential equations with infinite delay, J. Math. Anal. Appl., 338, 1340-1350 (2008).

[24] S. Liang, J.H. Zhang, Positive solutions for boundary value problems of nonlinear fractional differential equation, Nonlinear Anal., 71, 5545-5550 (2009).

[25] J. Henderson, A. Ouahab, Fractional functional differential inclusions with finite delay, Nonlinear Anal., 70, 2091-2105 (2009).

[26] B. Ahmad, J.J. Nieto, Existence of solutions for antiperiodic boundary value problems involving fractional differential equations via Leray-Schauder degree theory, Topol. Methods Nonlinear Anal., 35, 295-304 (2010).

[27] M.Q Feng, X.M. Zhang, W.G. Ge, New existence results for higher-order nonlinear fractional differential equation with integral boundary conditions, Bound. Value Probl., 2011, 120 (2011)

[28] B. Ahmad, J.J. Nieto, Sequential fractional differential equations with three-point boundary conditions, Comput. Math. Appl., 64, 3046-3052 (2012).

[29] Y.L Jiang, X. L. Ding, Waveform relaxation methods for fractional differential equations with the Caputo derivatives, J. Comput. Appl. Math., 238, 51-67 (2013).
[30] G. Wang, B. Ahmad, L. Zhang, R.P. Agarwal, Nonlinear fractional integro-differential equations on unbounded domains in a Banach space, J. Comput. App. Math., 249, 51-56 (2013).

[31] B. Ahmad, S.K. Ntouyas, Existence results for fractional differential inclusions arising from real estate asset securitization and HIV models, Advances in Difference Equations, 2013, (2013)

[32] R. Ponce, Hlder continuous solutions for fractional differential equations and maximal regularity, J. Differential Equations, 255, 3284-3304 (2013).

[33] H. Hejazi, T. Moroney, F. Liu, Stability and convergence of a finite volume method for the space fractional advectiondispersion equation, J. Comput. Appl. Math., 255, 684-697 (2014).

[34] X. Liu, Z. Liu, X. Fu, Relaxation in nonconvex optimal control problems described by fractional differential equations, J. Math. Anal. Appl., 409, 446-458 (2014).

[35] E. Ahmed, A.M. Elsayed, H.A.A. El-Saka, Equilibrium points, stability and numerical solutions of fractional-order predatorprey and rabies models, J. Math. Anal. Appl., 325, 542-553 (2007).

[36] Y. Yan, C. Kou. Stability analysis for a fractional differential model of HIV infection of $\mathrm{CD}^{+}$Tcells with time delay, Math. Comput. Simul., (2012), http://dx.doi.org/10.1016/j.matcom.2012.01.004.

[37] E. Ahmed, A.M. Elsayed, H.A.A. El-Saka, Equilibrium points, stability and numerical solutions of fractional-order predator-prey and rabies models, J. Math. Anal. Appl., 325, 542-553 (2007).

[38] E. Ahmed, A.S. Elgazzar, On fractional order differential equations model for nonlocal epidemics, Physica A, 379, 607-614 (2007).

[39] Y.S. Ding, H.P. Ye, A fractional-order differential equation model of HIV infection of $\mathrm{CD}^{+}{ }^{+} \mathrm{T}-$ cells, Math. Comput. Model., 50, 386-392 (2009).

[40] H.P. Ye, Y.S. Ding, Nonlinear dynamics and chaos in a fractional-order HIV model, Math. Probl. Eng. (2009), 12 pages, Article ID 378614, http://dx.doi.org/10.1155/2009/378614.

[41] D. Matignon, Stability result on fractional differential equations with applications to control processing, In: IMACS-SMC proceedings. Lille, France, 963-968 (1996).

[42] K. Diethelm, N. J. Ford, Analysis of fractional differential equations, J. Math. Anal. Appl., 265, 229-248 (2002).

[43] T. M. Atanackovic and B. Stankovic. An expansion formula for fractional derivatives and its application, Fract. Calc. Appl. Anal., 7, 365-378 (2004).

[44] T. M. Atanackovic and B. Stankovic, On a numerical scheme for solving differential equations of fractional order, Mech. Res. Commun., 35, 429-438 (2008). 


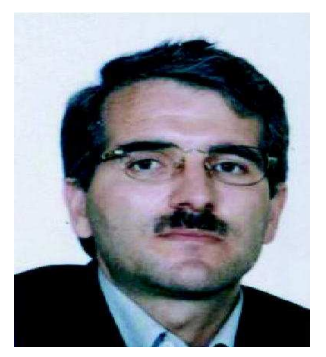

research articles in reputed international journals of applied mathematics.

M. Javidi received
the $\mathrm{PhD}$ degree in
Applied $\quad$ Mathematics at Iran University of Science \& Technology in Tehran. His research interests are in the areas of applied mathematics and numerical methods for fractional differential equations. He has published

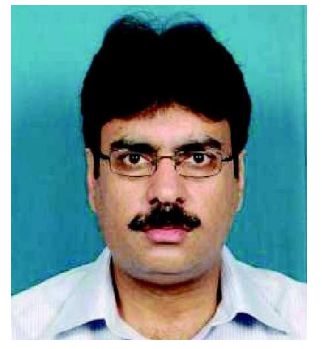

Bashir Ahmad is Full Professor of Mathematics at King Abdulaziz University, Jeddah, Saudi Arabia. $\mathrm{He}$ received his Ph.D. degree from Quaid-i-Azam University, Islamabad, Pakistan in 1995. His research interest includes approximate/numerical

methods for nonlinear problems involving a variety of differential equations, existence theory of fractional differential equations, stability and instability properties of dynamical systems, impulsive systems, and scattering theory. He has published extensively on nonlinear boundary value problems. He was honored with "Best Researcher of King Abdulaziz University" award in 2009. $\mathrm{He}$ is a reviewer of several international journals. He is Assistant Managing Editor of Bulletin of Mathematical Sciences (Springer) and member of editorial boards of several journals. 